Article

\title{
Protective Effect of Resveratrol on Biomarkers of Oxidative Stress Induced by Iron/Ascorbate in Mouse Spermatozoa
}

\section{María Angélica Mojica-Villegas ${ }^{1}$, Jeannett Alejandra Izquierdo-Vega ${ }^{2}$, Germán Chamorro-Cevallos ${ }^{1}$ and Manuel Sánchez-Gutiérrez ${ }^{2, *}$}

1 Preclinical Toxicology Laboratory, Department of Pharmacy, National School of Biological Sciences, National Polytechnic Institute, Mexico City 07510, Mexico;

E-Mails: moviangel13@yahoo.com.mx (M.A.M.-V.); gchamcev@yahoo.com.mx (G.C.-C.)

2 Academic Area of Medicine, Institute of Health Sciences at Autonomous University of the State of Hidalgo, Pachuca, Hidalgo 11510, Mexico; E-Mail: jizquierdovega@gmail

* Author to whom correspondence should be addressed; E-Mail: spmtz68@yahoo.com.mx; Tel.: +52-771-7172000 (ext. 4308); Fax: +52-771-7172000 (ext. 5111).

Received: 25 November 2013; in revised form: 27 December 2013 / Accepted: 17 January 2014 / Published: 27 January 2014

\begin{abstract}
Resveratrol (RVT) is a polyphenolic compound found mainly in the grape and attributed with various pharmacological properties, among them their antioxidant activity. In the present study, we assess the antioxidant activity of resveratrol on oxidative damage induced by ferrous iron/ascorbate $(100 \mu \mathrm{M} / 150 \mu \mathrm{M})$ in sperm of $\mathrm{CD}^{+}{ }^{+}$mice. We evaluated several parameters in spermatozoa treated with or without resveratrol: (i) sperm quality analysis; (ii) mitochondrial transmembrane potential $\left(\Delta \psi_{\mathrm{m}}\right)$; (iii) ROS generation; (iv) superoxide dismutase (SOD) activity; (v) glutathione peroxidase (GPX) activity; (vi) lipid peroxidation; (vii) and in vitro fertilization (IVF) capability. Spermatozoa treated with RVT $(15 \mu \mathrm{g} / \mathrm{mL})$ before ferrous iron/ascorbate treatment exhibited: a significant increase in motility (8-fold), a significant increase in viability (2-fold), a significant increase in $\Delta \psi_{\mathrm{m}}$ (1.15-fold), accompanied with a significant decrease in the generation of ROS (4.96-fold), a significant decrease in GPX activity (1.32-fold), and a significant decrease in lipid peroxidation concentration (10.29-fold) relative to spermatozoa treated with ferrous iron/ascorbate; however, no changes in SOD activity were observed. Finally, spermatozoa treated with RVT before ferrous iron/ascorbate treatment showed a significant increase in oocyte fertilization (1.2-fold), relative to spermatozoa treated with ferrous iron/ascorbate. These results suggest that RVT possesses antioxidant properties that may
\end{abstract}


prevent the deleterious effects produced by oxidative damage on spermatozoa, resulting in the maintenance of fertility.

Keywords: resveratrol; spermatozoa; oxidative stress; in vitro fertilization; mitochondrial transmembrane potential

\section{Introduction}

Oxidative stress is defined as an imbalance between the formation of reactive oxygen species (ROS, including hydroxyl radicals, superoxide anions and hydrogen peroxide) and the antioxidant capacity [1]. Spermatozoa are particularly susceptible to oxidative stress due to the high membrane content of polyunsaturated fatty acid and the intracellular deficiency of antioxidant enzymes [2].

Low ROS levels are required for the regulation of the principal functions of sperm. Such functions are capacitation, acrosome reaction and fertilizing ability [3]. However, when levels are excessive, ROS attack polyunsaturated fatty acids in the sperm plasma membrane, leading to lipid peroxidation [4]. An imbalance between ROS generation and the antioxidant capacity of spermatozoa leads to oxidative stress, and the exposure of spermatozoa to this condition appears to be related to male infertility $[3,5]$.

Seminal plasma and sperm are endowed with an array of antioxidant enzymes that act as free radical scavengers to protect spermatozoa against cellular damage caused by oxidative stress [6]. Such enzymes include glutathione peroxidase, glutathione reductase, superoxide dismutase, catalase, low molecular weight antioxidants, vitamin E, and vitamin C. Numerous attempts have been made to avoid or minimize oxidative stress in sperm by the use of antioxidants. However, the effectiveness of this strategy is still being debated [6,7].

A great variety of substances are antioxidants, including vitamins, enzymes and other free radical scavengers. Resveratrol (RVT) (trans-3,5,4'-trihydroxystilbene) is a polyphenol compound present in grapes, peanuts, berries and wine [8]. It is a phytoalexin whose biological function is to protect the plant in case of parasitic attack or environmental stress [9]. The literature contains numerous reports on the wide variety of properties of RVT, including its anti-inflammatory, cardioprotective, anticancer, antimicrobial, anti-aging and antioxidant effects [10]. This biological activity is carried out by a wide variety of mechanisms, one of the most important of which is antioxidant activity due to free radical scavenging [11]. Some recent studies in animal models demonstrate that RVT has positive effects on the hypothalamic-pituitary-gonad axis, blood testosterone levels, sperm production and sperm motility [12,13]. Furthermore, RVT may decrease germ cell apoptosis [14,15]. Despite these insights, the effect of RVT supplementation on male infertility has not yet been explored.

The aim of the present study was to explore the antioxidant activity of RVT against oxidative stress induced in mouse spermatozoa by exposure to ferrous iron/ascorbate in vitro. 


\section{Experimental Section}

\subsection{Chemicals}

Resveratrol, bovine serum albumin fraction V (BSA), butylated hydroxytoluene (BHT), human chorionic gonadotropin (hCG), lactic acid, sodium pyruvate, formaldehyde, thiobarbituric acid (TBA) and trypan blue were purchased from Sigma Chemical Co. (St Louis, MO, USA). Gonadotropin (PMSG) from pregnant mare serum was acquired from Intervet, International B.V. (Boxmeer, Holland). The mito Probe JC-1 assay kit and an ROS detection reagent were from Molecular Probes Invitrogen (Mount Waverley, Australia), trichloroacetic acid (TCA) from J.T. Baker (Pillipsburg, NJ, USA), and the RANSOD and RANSEL kits from RANDOX (Crumlin, UK).

\subsection{Animals}

Animals were maintained according to the norms of the Institutional Ethics Animal Care and Use Committee (CIECUAL), and in compliance with the Guidelines for Use and Care of Laboratory Animals. Male sexually mature $(30 \pm 2 \mathrm{~g})$ and female immature $\mathrm{CD}^{+}$mice (5 old weeks) were obtained from the Institute of Health Sciences at the Autonomous University of Hidalgo (Hidalgo, Mexico). Animals were maintained under standard conditions, with a $12 \mathrm{~h} / 12 \mathrm{~h}$ light/dark cycle, constant temperature $\left(22 \pm 2{ }^{\circ} \mathrm{C}\right)$ and humidity $(50 \%)$, and food and water freely available in their home cages.

\subsection{Experimental Design}

After animal sacrifice by cervical dislocation, testes-epididymis-vas deferent complexes were dissected. Spermatozoa were collected by flushing vas deferens and cauda epididymis lumens with $1 \mathrm{~mL}$ of Media M-16 (100 mM NaCl, $25 \mathrm{mM} \mathrm{NaHCO}_{3}, 5.5 \mathrm{mM}$ glucose, $2.6 \mathrm{mM} \mathrm{KCl}, 1.56 \mathrm{mM} \mathrm{Na}_{2} \mathrm{HPO}_{4}$, $0.5 \mathrm{mM}$ sodium pyruvate, $1.8 \mathrm{mM} \mathrm{CaCl}_{2}, 0.5 \mathrm{mM}, \mathrm{MgCl}_{2}$ and $20 \mathrm{mM}$ sodium lactate, $\left.\mathrm{pH} 7.4\right)$ at $37^{\circ} \mathrm{C}$. All medium were prepared with deionized water (Milli-Q Plus water system, Millipore). The spermatozoa were allowed to swim in the medium for $10 \mathrm{~min}$ at $37^{\circ} \mathrm{C}$. The sperm suspension was then transferred into a plastic tube and the motility and concentration of sperm were determined. Samples were used only if at least $80 \%$ of total sperm were motile and the concentration was at least $10 \times 10^{6} \mathrm{sperm} / \mathrm{mL}$.

The sperm suspensions from three animals were pooled and divided into four experimental groups: (1) spermatozoa without treatment (Control); (2) spermatozoa treated with $15 \mu \mathrm{g} / \mathrm{mL}$ RVT during $15 \mathrm{~min}$; (3) spermatozoa treated with $100 \mu \mathrm{M} / 150 \mu \mathrm{M}$ ferrous iron/ascorbate for $45 \mathrm{~min}$; and (4) spermatozoa treated with $15 \mu \mathrm{g} / \mathrm{mL}$ RVT during $15 \mathrm{~min}$ before treatment with $100 \mu \mathrm{M} / 150 \mu \mathrm{M}$ ferrous iron/ascorbate for $45 \mathrm{~min}$. After incubation, the following parameters were evaluated in the four groups: (i) sperm quality; (ii) mitochondrial transmembrane potential $\left(\Delta \psi_{\mathrm{m}}\right)$; (iii) ROS generation; (iv) superoxide dismutase activity (SOD); (v) glutathione peroxidase (GPX) activity; (vi) lipid peroxidation; and (vii) in vitro fertilization (IVF) capability. For each parameter, three independent experiments were performed in duplicate. 


\subsection{Spermatozoa Quality}

Sperm parameters, including viability and motility, were evaluated according to a previously described method [16]. Spermatozoa motility (percent motile cells) was assessed in ten random fields using a phase contrast microscope (Carl Zeiss, Germany) at 400X. Sperm concentration was measured in a hemocytometer and expressed as one million $/ \mathrm{mL}$ of suspension. Spermatozoa viability was determined by trypan blue exclusion assay. For each sample, 100-200 cells were counted.

\subsection{Mitochondrial Membrane Potential $\left(\Delta \psi_{m}\right)$}

Spermatozoa $\Delta \psi_{\mathrm{m}}$ was measured using a spectrofluorometer (Perkin Elmer LS 55, Norwalk, CT, USA) and a MitoProbe JC-1 assay kit. JC-1 is a lipophilic cation that differentially labels mitochondria on the basis of membrane potential. After incubating a solution of $5 \times 10^{6}$ spermatozoa $/ \mathrm{mL}$ with one of the treatments, two micro liters of JC-1 were added. High membrane potential was associated with emission at $590 \mathrm{~nm}$ (red), and low membrane potential with emission at $530 \mathrm{~nm}$ (green) when spermatozoa were excited at $488 \mathrm{~nm}$. The $\Delta \psi \mathrm{m}$ was determined by a ratio of fluorescence intensity at $590 \mathrm{~nm}$ and at $530 \mathrm{~nm}$.

\subsection{ROS Level in Spermatozoa}

ROS generation was evaluated using (5-(and-6)-chloromethyl-2',7'-dichlorofluorescein diacetate and acetyl ester (CM- $\mathrm{H}_{2}$ DCFDA). The method is based on the ROS-dependent oxidation of $2^{\prime}, 7^{\prime}$-dichlorofluorescin diacetate to fluorescent dichlorofluorescein (Invitrogen Molecular Probes). Spermatozoa $\left(5 \times 10^{6} / \mathrm{mL}\right)$ were treated with $10 \mu \mathrm{M} \mathrm{CM}-\mathrm{H}_{2}$ DCFDA for $30 \mathrm{~min}$ at $37{ }^{\circ} \mathrm{C}$ in the dark. Fluorescence intensities were measured using a spectrofluorometer (Perkin Elmer LS 55, Norwalk, CT, USA).

\subsection{SOD Activity in Spermatozoa}

Enzymatic activity of SOD was measured using the RANSOD assay kit (RANDOX). This method uses xanthine oxidase to generate superoxide radicals, which react with 2-(4-iodophenil)-3-(4nitrophenol)-5-phenyltetrazolium chloride to form a red formazan dye. SOD activity was measured at $505 \mathrm{~nm}$ with a spectrophotometer (Power Wave Xs, Biotek Instruments Inc., Highland Park, Winooski, VT, USA), based on evaluating the degree of inhibition of xanthine to water and molecular oxygen. $10 \times 10^{6}$ Cells were treated with 1:1 $0.1 \%$ triton X 100-PBS for $20 \mathrm{~min}$. The cells were then centrifuged at $5000 \mathrm{rpm}$ at $4{ }^{\circ} \mathrm{C}$ for $10 \mathrm{~min}$. Finally, the supernatant was obtained for determining SOD activity.

\subsection{GPX Activity in Spermatozoa}

Enzymatic activity of GPX was measured using the RANSEL assay kit (RANDOX). GPX catalyzes glutathione oxidation (GSH) by cumene hidroperoxide. Oxidized glutathione (GSSG) in the presence of glutathione reductase (GR) and NADPH is immediately changed to the reduced form with a concomitant oxidation of $\mathrm{NADP}^{+}$. GPX activity was measured as the decrease of absorbance at $340 \mathrm{~nm}$ 
with a spectrophotometer (Power Wave Xs, Biotek Instruments Inc., Highland Park, Winooski, VT, USA). $10 \times 10^{6}$ Cells were treated with $1: 10.1 \%$ triton $\mathrm{X} 100$-PBS for $20 \mathrm{~min}$. The cells were then centrifuged at $5000 \mathrm{rpm}$ at $4{ }^{\circ} \mathrm{C}$ for $10 \mathrm{~min}$. Finally, the supernatant was obtained for determining GPX activity.

\subsection{TBARS Concentration in Spermatozoa}

Malondialdehyde (MDA), other aldehydes and lipid hydroxyperoxides are able to form adducts with TBA (Sigma-Aldrich Co., St. Louis, MO, USA). The MDA concentration was used as an index of lipid peroxidation using the thiobarbituric acid reactive substances (TBARS) method [17]. Briefly, sperm suspensions $\left(10 \times 10^{6}\right.$ cells $\left./ \mathrm{mL}\right)$ were added to a mixture containing $0.5 \%$ TBA and $3.75 \%$ BHT in methanol. Samples were heated in a boiling water bath for $30 \mathrm{~min}$ and then cooled. Absorbance was then measured at $532 \mathrm{~nm}$ with a spectrophotometer (Power Wave Xs, Biotek Instruments Inc., Winooski, VT, USA).

\subsection{In Vitro Fertilization (IVF)}

\subsubsection{Egg Recovery}

Eggs were obtained from immature female $\mathrm{CD}^{+}$mice. Super ovulation was induced by intraperitoneal injection of 10 IU Pregnant mare's serum gonadotropin (PMSG), and $48 \mathrm{~h}$ later of $10 \mathrm{IU}$ human chorionic gonadotropin (hCG). Approximately 14-16 h after the hCG injection, animals were sacrificed by cervical dislocation. The uterine ovary-salpinge-horn complex was dissected and suspended in M-16 medium. In each oviduct, the ampulla were punctured, and the cumulus-egg complex was extruded and placed in a solution of $0.1 \%(\mathrm{w} / \mathrm{v})$ hyaluronidase with $\mathrm{M}-16$ medium for seven min at $37{ }^{\circ} \mathrm{C}$ to remove cumulus cells. Then, cumulus free eggs were pooled and washed with M-16 medium to remove hyaluronidase. The fertilization assay was carried out only with mature eggs showing a polar body and with the zone intact.

\subsubsection{IVF Assay}

For each experimental group 40 random eggs in $200 \mu \mathrm{L}$ M-16 medium were placed on a standard slide with two polished spherical depressions of approximately 0.5 to $0.8 \mathrm{~mm}$ deep (VWR International). The eggs were then inseminated with $10 \mu \mathrm{L}\left(1 \times 10^{5}\right.$ cells $)$ of one of four sperm suspensions (see Section 2.3). In each case, the sperm suspension had been previously capacitated in M-16 medium (supplemented with $4 \mathrm{mg} / \mathrm{mL} \mathrm{BSA}$ ) for $1 \mathrm{~h}$ at $37{ }^{\circ} \mathrm{C}$. Gametes were co-incubated during $24 \mathrm{~h}$ at $37{ }^{\circ} \mathrm{C}$ in a high-humidity incubator under an atmosphere of $5 \% \mathrm{CO}_{2} / 95 \%$ air. The embryos were examined for the presence of two to six cells as an indication of successful fertilization. The cells were fixed in $3 \%$ formaldehyde-PBS (v/v) and observed by phase contrast microscopy. Three independent experiments with different batches of cells were performed. 


\subsection{Data Analysis}

Statistical analysis was carried out with analyses of variance (ANOVA) followed by a Bonferonni correction to evaluate pair wise differences. A $p$-value $<0.05$ was considered significant. Data are expressed as the means \pm standard deviation (SD). All analyses were performed using the statistical software Stata 8.0 (Stata Corp., College Station, TX, USA).

\section{Results}

\subsection{Effect of RVT on Spermatozoa Quality}

Ferrous iron/ascorbate $(100 \mu \mathrm{M} / 150 \mu \mathrm{M})$ was used to induce oxidative stress and cause deleterious effects on spermatozoa quality. The results show a significant reduction $(p<0.001)$ in motility (11.0 fold) and viability (4.39 fold). On the other hand, pretreatment with $15 \mu \mathrm{g} / \mathrm{mL}$ of RVT for $15 \mathrm{~min}$ provided protection of spermatozoa. Compared to the spermatozoa treated only with iron/ascorbate, the RVT pretreated spermatozoa showed an 8.0 fold increase in motility and a 2.0 fold increase in viability (Table $1 ; p<0.001$ ). Regarding both parameters, there was a significant enhancement of spermatozoa quality.

Table1. Spermatozoa quality.

\begin{tabular}{ccccc}
\hline Sperm parameters & Control & RVT & Ferrous iron/ascorbate & RVT + Ferrous iron/ascorbate \\
\hline Motility (\%) & $73.3 \pm 5.7$ & $73.3 \pm 2.8$ & $6.66 \pm 2.8 *$ & $53.3 \pm 5.7 * *$ \\
Viability (\%) & $65.0 \pm 8.6$ & $59.6 \pm 6.1$ & $14.8 \pm 3.3 *$ & $30.5 \pm 6.5 * *$ \\
\hline
\end{tabular}

Values represent the mean $\pm \mathrm{SD} ; * p<0.001$ vs. control group; $* * p<0.001 v s . \mathrm{Fe}^{2} /$ asc group.

\subsection{Protective Effect of Resveratrol on Mitochondrial Function}

The evaluation of mitochondrial membrane potential $\left(\Delta \psi_{\mathrm{m}}\right)$ is a reliable indicator of sperm quality. We evaluated mitochondrial activity using fluorophore JC-1. Compared with the control group, the ferrous iron/ascorbate treatment significantly reduced the $\Delta \psi_{\mathrm{m}}$ of spermatozoa (1.5 fold). Contrarily, compared to treatment with ferrous iron/ascorbate alone, pretreatment with RVT followed by exposure to ferrous iron/ascorbate resulted in spermatozoa with a higher $\Delta \psi_{\mathrm{m}}(1.15$ fold; $p<0.05$; Figure 1). Interestingly, spermatozoa treated only with RVT showed a significant decrease in the $\Delta \psi_{\mathrm{m}}$ (1.13 fold) compared with the control group.

\subsection{Resveratrol Diminished Intracellular ROS Generation}

ROS generation was measured by fluorescence using the reactive oxygen species detection reagent. Compared with the control, there was a significantly greater level of ROS (1.75 fold) in spermatozoa treated only with ferrous iron/ascorbate (Figure 2). On the other hand, compared to spermatozoa treated only with ferrous iron/ascorbate, spermatozoa pretreated with RVT and then exposed to ferrous iron/ascorbate had a significant lower level of ROS (4.96 fold; $p<0.001)$. 
Figure 1. Effect of RVT on $\Delta \psi_{\mathrm{m}}$. Spermatozoa were obtained from ductus deferens in M-16 medium, pooled and incubated with $\mathrm{RVT}, \mathrm{Fe}^{2+} /$ asc or $\mathrm{RVT}+\mathrm{Fe}^{2+} /$ asc. $\Delta \psi_{\mathrm{m}}$ was determined using the Mito Probe JC-1 assay kit and the fluorescence was measured on a spectrofluorometer. Values represent the mean $\pm \mathrm{SD}$ of three independent experiments performed in duplicate. ${ }^{*} p<0.05$ vs. control group. ** $p<0.05 v s$. $\mathrm{Fe}^{2+} /$ asc group.

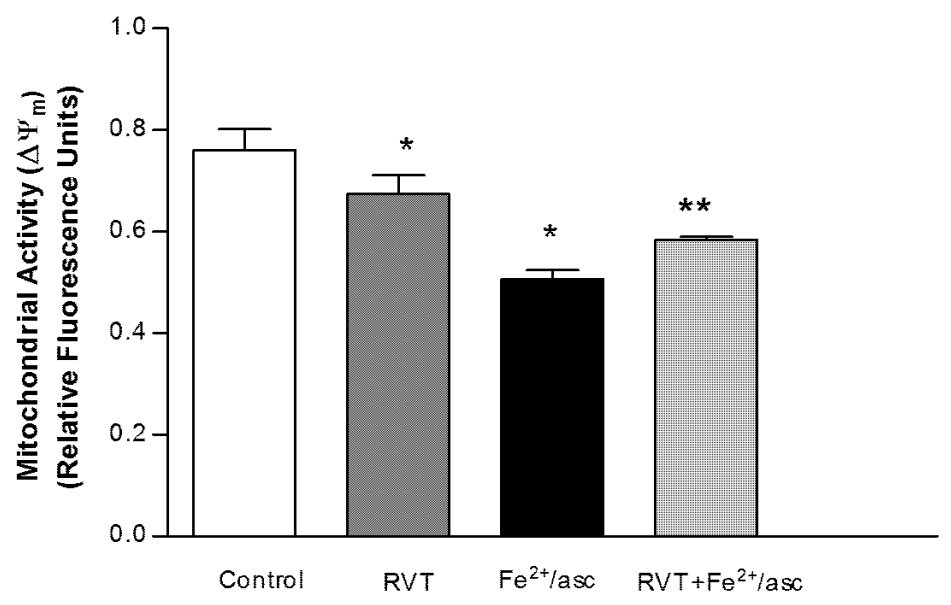

Figure 2. Effect of RVT treatment on intracellular ROS levels. Spermatozoa were obtained from ductus deferens in M-16 medium, pooled and incubated with $\mathrm{RVT}, \mathrm{Fe}^{2+} / \mathrm{asc}$ or $\mathrm{RVT}+\mathrm{Fe}^{2+}$ /asc. ROS levels were evaluated using the $\mathrm{CM}-\mathrm{H}_{2} \mathrm{DCFDA}$ reagent. Fluorescence was measured in a spectrofluorometer. Values represent the mean $\pm \mathrm{SD}$ of three independent experiments performed in duplicate. ${ }^{*} p<0.001$ vs. control group. ${ }^{*} * p<0.001$ vs. $\mathrm{Fe}^{2+}$ asc group.

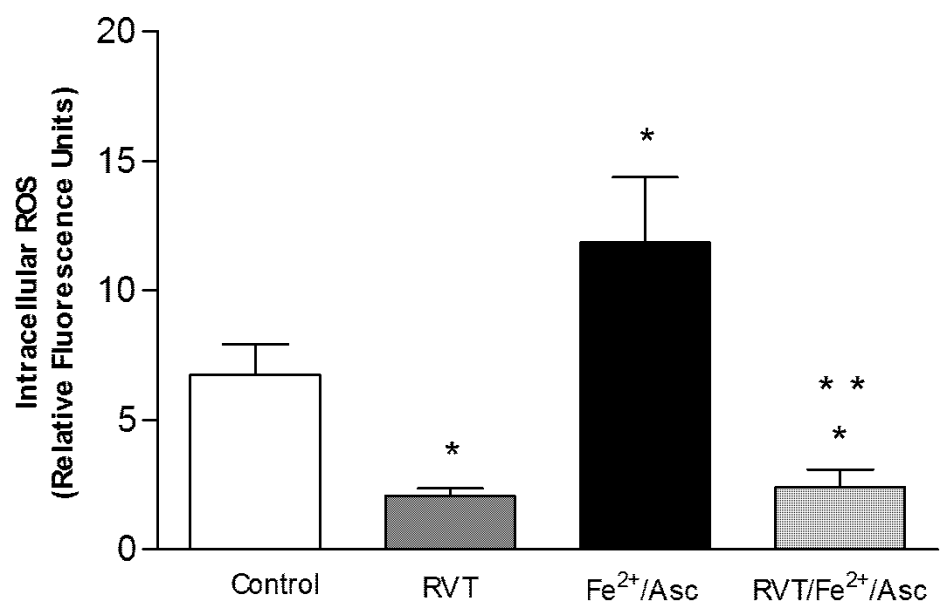

\subsection{Effect of RVT on SOD and GPX Activity}

SOD and GPX activity was analyzed in the spermatozoa of all treatments groups to evaluate oxidative stress. Compared with the control, treatment only with ferrous iron/ascorbate led to a significantly greater GPX activity in spermatozoa (1.76 fold; Figure 3). Contrarily, compared to treatment only with ferrous iron/ascorbate, pretreatment with RVT followed by exposure to ferrous iron/ascorbate resulted in significantly lower GPX activity (1.32 fold). No significant differences were found in SOD activity between any of the groups (Figure 4). 
Figure 3. Effect of RVT on GPX activity. Spermatozoa were obtained from ductus deferens in M-16 medium, pooled and incubated with $\mathrm{RVT}, \mathrm{Fe}^{2+} / \mathrm{asc}$ or $\mathrm{RVT}+\mathrm{Fe}^{2+} / \mathrm{asc}$. Activity of GPX was measured with a spectrophotometer after using the RANSEL assay kit. Values represent the mean \pm SD of three independent experiments performed in duplicate. $* p<0.05$ vs. control group. $* * p<0.05 v s$. $\mathrm{Fe}^{2+} /$ asc group.

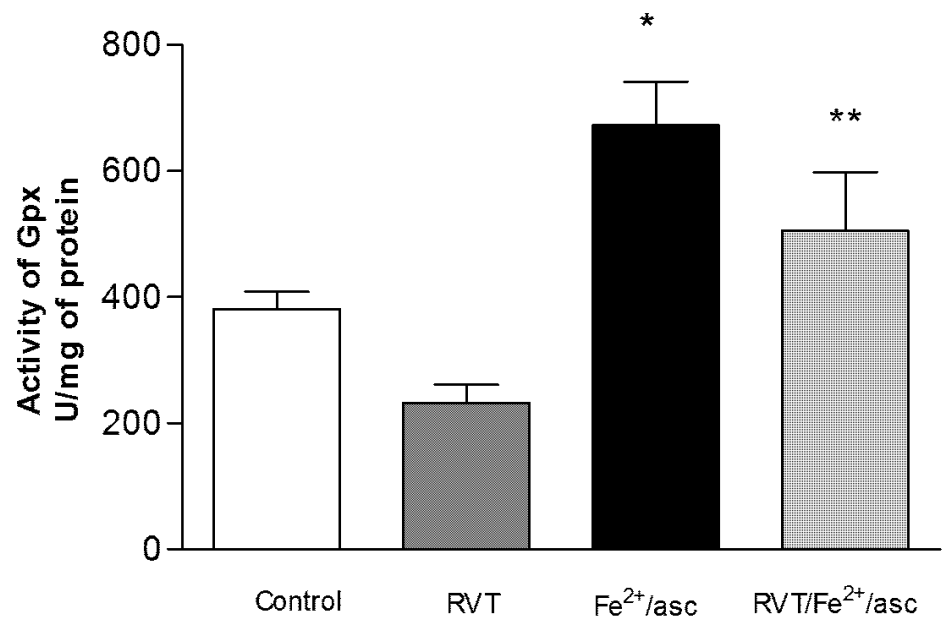

Figure 4. Effect of RVT on SOD activity. Spermatozoa were obtained from ductus deferens in M-16 medium, pooled and incubated with RVT, $\mathrm{Fe}^{2+} /$ asc or $\mathrm{RVT}+\mathrm{Fe}^{2+} /$ asc. Enzymatic activity of SOD was measured with a spectrophotometer after using the RANSOD assay kit. Values represent the mean \pm SD of three independent experiments performed in duplicate. No significant differences were observed.

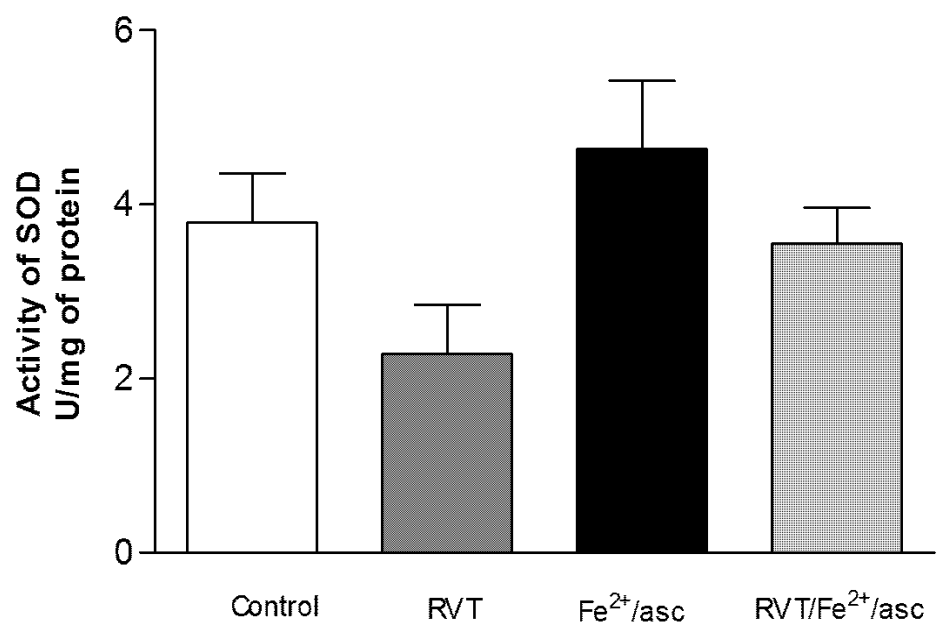

\subsection{Effect of RVT on Lipid Peroxidation}

Lipid peroxidation was assessed as a marker of oxidative damage (Figure 5). Compared to the control, spermatozoa treated only with ferrous iron/ascorbate showed a significantly higher content of MDA (13.38 fold). In contrast, compared to treatment only with ferrous iron/ascorbate, pretreatment with RVT followed by exposure to ferrous iron/ascorbate resulted in a significant reduction in the MDA content (10.29 fold) of spermatozoa $(p<0.001)$. 
Figure 5. Effect of RVT on lipid peroxidation. Spermatozoa were obtained from ductus deferens in M-16 medium, pooled and incubated with $\mathrm{RVT}, \mathrm{Fe}^{2+} / \mathrm{asc}$ or $\mathrm{RVT}+\mathrm{Fe}^{2+} / \mathrm{asc}$. Lipid peroxidation was measured with a spectrophotometer using the TBARS method. Values are the mean $\pm \mathrm{SD}$ of three independent experiments performed in duplicate. $* p<0.001 v s$. control group. ** $p<0.001 v s$. $\mathrm{Fe}^{2+} /$ asc group.

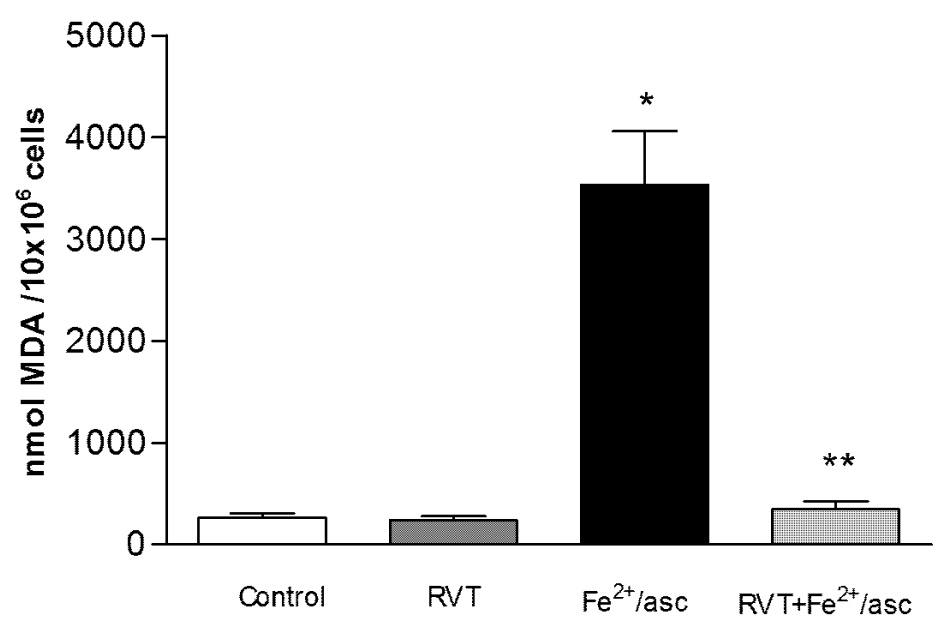

\subsection{Resveratrol Retains in Vitro Fertilization Capability}

Compared to untreated (control) and RVT-only treated spermatozoa, a significantly lower percentage of these cells treated only with ferrous iron/ascorbate had the capacity to fertilize oocytes (78.66\% and $76.66 \%$ vs. 61\%, respectively; Figure 6). On the other hand, compared to treatment only with ferrous iron/ascorbate, pretreatment with RVT followed by exposure to ferrous iron/ascorbate resulted in a significantly greater percentage of spermatozoa with the capacity to fertilize oocytes (74\% vs. 61\%; $p<0.001)$.

Figure 6. Effect of RVT on IVF capability. Spermatozoa from the different groups were incubated with eggs during $24 \mathrm{~h}$ at $37{ }^{\circ} \mathrm{C}$ in an incubator under an atmosphere of $5 \% \mathrm{CO}_{2} / 95 \%$ air. Eggs with 2 to 6 cells were considered fertilized. Values represent the mean $\pm \mathrm{SD}$ of three independent experiments performed in duplicate. ${ }^{*} p<0.001 v s$. control group. $* * p<0.001$ vs. $\mathrm{Fe}^{2+}$ asc group.

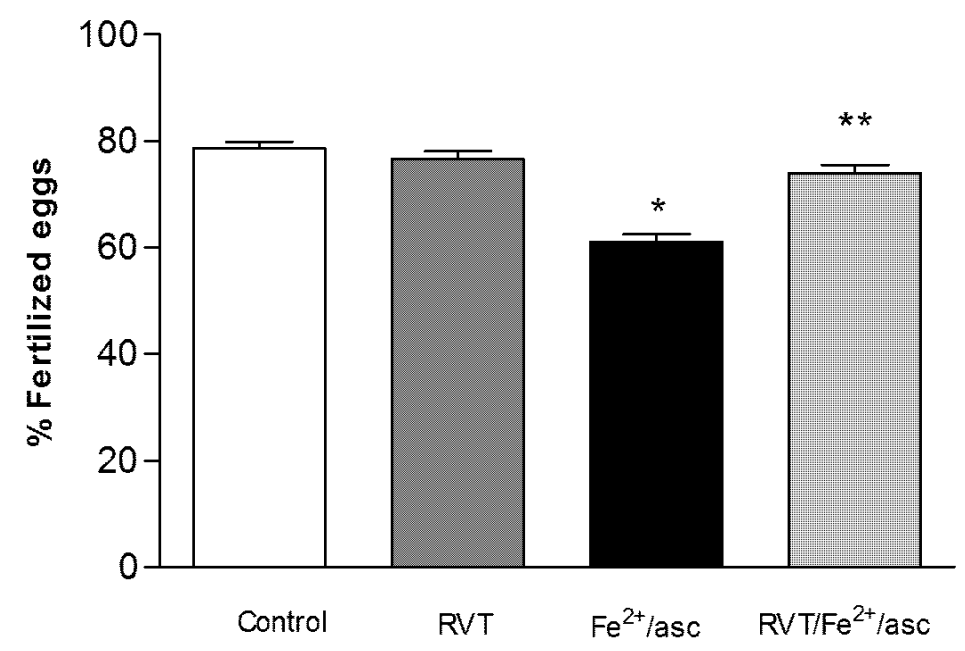




\section{Discussion}

Oxidative stress is defined as an imbalance between ROS generation and antioxidant capacity. As mentioned previously, spermatozoa are highly vulnerable to attack from ROS due to their low cytoplasmic antioxidant capacity and high membrane polyunsaturated fatty acid content [18].

Whereas the generation of low levels of ROS is an important component of the signal transduction stimulating capacity of spermatozoa [19], excessive ROS levels induce lipid peroxidation and reduce the ionophore-induced acrosome reaction [20]. Only acrosome-reacted sperm are able to penetrate the pellucida zone of the oocyte [21].

The possible relationship of oxidative stress in spermatozoa to male infertility is supported by both experimental and epidemiological evidence [3,5]. High ROS concentrations lead to pathological changes in spermatozoa by intensifying the peroxidation of lipids found in the plasma membrane of these cells, which in turn impairs the fusion of the plasma of the male and female gametes [3]. Thus excessive ROS levels affect basic parameters of sperm, such as sperm count, morphology and motility [22]. ROS is also able to stimulate the oxidation of the sulfhydryl radical as well as the DNA of molecules. Moreover, DNA fragmentation (an indicator of nuclear structural integrity) was shown to correlate strongly with ROS concentration [23]. Further evidence of the damage caused to spermatozoa by excessive levels of ROS is provided by the fact that levels of DNA oxidation in sperm are higher in infertile than fertile men [24].

The use of antioxidant supplementation with vitamins $\mathrm{E}$ and $\mathrm{C}, \mathrm{GSH}$, catalase, selenium or SOD has been a strategy for the treatment of oxidative stress. However, results have caused controversy over the effectiveness of this strategy for improving fertility [25,26]. Recently, some studies have reported a dose-response relationship between the daily intake of antioxidant nutrients (carotenoids, lycopene and lutein) and semen quality [27,28]. In addition, an inverse relationship has been shown between the intake of whole dairy foods (with the fat content intact) and semen quality in young men [29].

Consequently, there is great interest in evaluating antioxidant agents that could protect spermatozoa from ROS. One possible agent, resveratrol (RVT), is a natural phytoalexin with antioxidant properties. It is widely consumed in the Mediterranean diet in the form of peanuts, grapes and wine [8]. Assays with in vivo experimental models have shown a positive RVT-induced effect on male reproduction, reported as the enhancement of testosterone levels, motility and sperm count [13]. Moreover, it has recently been shown that in vivo treatment with RVT prevents oxidative stress in testes of hyperthyroid rats [30] and rats treated with a chemotherapy drug [31]. However, the antioxidant mechanism of action of RVT on male reproduction is as yet unknown. The aim of the present study was to carry out an in vitro analysis of the protective effect of RVT against oxidative damage caused by ferrous iron/ascorbate on mouse spermatozoa.

The daily intake of RVT in the Mediterranean diet is approximately $0.02 \mathrm{mg} / \mathrm{kg}$, assuming that red wine is the main dietary source and the average concentration of trans-RVT in wine is $5 \mathrm{mg} / \mathrm{L}[32,33]$. Recent in vivo studies have shown that repeated doses of RVT at $10 \mathrm{mg} / \mathrm{kg}, 20 \mathrm{mg} / \mathrm{kg}$ and $50 \mathrm{mg} / \mathrm{kg}$, being 500, 1000, 2500 times higher than the aforementioned average consumption, respectively, provides a sufficiently large safety margin [34] and protects testes and spermatogenesis against oxidative stress [13,30,31]. Unfortunately, RVT concentrations in testes of animals receiving chronic 
treatment with this polyphenol are unknown. Additionally, the low plasmatic levels of RVT attained $(<40 \mathrm{nM})$ in such animals would be inadequate for eliciting a biological effect, considering the quantity needed for activity in cells with in vitro concentrations of 5-100 $\mu \mathrm{M}$ [35]. Based on an evaluation of motility of spermatozoa with different concentrations of RVT (data not shown), $15 \mu \mathrm{g} / \mathrm{mL}$ was the dose of this compound chosen for the present study. In accordance with this decision, an antioxidant effect on spermatozoa has been found with RSV treatment when employing concentrations in the range 5 to $20 \mu \mathrm{g} / \mathrm{mL}$ [36].

Several studies have demonstrated that ferrous iron/ascorbate causes oxidative damage in the spermatozoa of various animal species [37,38]. The ferrous iron/ascorbate system generates the hydroxyl radical through the Haber-Weiss or Fenton reaction [39]. High levels of ROS are associated with a reduction in motility and ATP levels of spermatozoa, which is attributed to mechanisms independent of oxidative phosphorylation that can lead to the inactivation of glycolytic enzymes [40]. In the present study we observed that the diminution in quality (motility and viability) of sperm induced by the pro-oxidant ferrous iron/ascorbate was prevented by RVT, suggesting antioxidant activity by the latter compound. There is evidence that RVT is an effective scavenger of the hydroxyl radical and superoxide anion, as well as other radicals induced by metals [41].

Spermatozoon motility has been associated with the functional status of mitochondria because motility is ATP-dependent. Since mitochondrial membrane potential $\left(\Delta \psi_{\mathrm{m}}\right)$ is widely used to assess the functional status of mitochondria [42], we evaluated this parameter in spermatozoa. Results show that RVT pretreatment protected against the decrease in $\Delta \psi_{\mathrm{m}}$ caused when spermatozoa are treated only with ferrous iron/ascorbate. Additionally, we found that treatment only with RVT significantly decreased $\Delta \psi_{\mathrm{m}}$ without any change in motility. Consistent with this observation, it has been shown that $\Delta \psi_{\mathrm{m}}$ decreased in a concentration-dependent manner with increasing concentrations of RVT (including the same concentration used in this study), without affecting motility, acrosome integrity or plasma membrane integrity in ram spermatozoa [36]. Likewise, a decrease in the $\Delta \psi_{\mathrm{m}}$ of neuroblastoma cells was found as a result of RVT treatment in a concentration-dependent manner [43].

Concerning the effects of RVT on mitochondrial bioenergetics, it has been shown that this compound inhibits the brain mitochondrial respiratory chain of complex III, thus preventing the production and inducing the scavenging of ROS [44]. The present study shows that RVT prevents the generation of intracellular ROS induced by the pro-oxidant ferrous iron/ascorbate in spermatozoa, suggesting the antioxidant property of RVT as an ROS scavenger. This protective role is further evidenced by the enzymatic activity of GPx in spermatozoa. GPx catalyzes the reduction of peroxide by oxidizing glutathione. We found that RVT prevents the increase in GPx activity that results from exposure to ferrous iron/ascorbate.

On the other hand, we did not find any significant difference in SOD activity with RVT pretreatment. SOD catalyzes the dismutation of $\mathrm{O}_{2}{ }^{-}$into oxygen and hydrogen peroxide. The lack of difference with and without RVT pretreatment may be due to the fact that the pro-oxidant ferrous iron/ascorbate generates the hydroxyl radical through the Haber-Weiss or Fenton reaction [40].

Oxidative damage is considered the main indicator of a loss of cellular function caused by oxidative stress [1]. Hence, evaluation of the TBARS concentration was carried out with the four groups of the present study. The results indicate that there is oxidative damage to spermatozoa membranes caused by ferrous iron/ascorbate, as well as protection against such damage by RVT pretreatment. In accordance 
with these results, previous studies have reported that RVT prevents the lipid peroxidation induced by tert-butyl hydroperoxide in human sperm [45], and that RVT is a lipophilic molecule that prevents lipid peroxidation induced by Fenton reaction products $[44,46]$. The protective effects of RVT against oxidative damage might be attributed to a hydrogen electron donation from its hydroxyl groups [47].

To further explore the protective role of RVT against the oxidative stress in spermatozoa caused by ferrous iron/ascorbate, we evaluated the IVF capacity of the four groups, finding that RVT pretreatment protected the IVF process. The results of various reports are consistent with this observation. For example, it has been reported that the oxidative damage induced by ferrous iron/ascorbate leads to a decrease in cholesterol efflux in macrophages, and that this decrease is restored by RVT treatment in a concentration-dependent manner [46]. It is also known that enhanced cholesterol efflux is involved in improving sperm capacitation and IVF capacity [48]. One antioxidant, glutathione, has recently been shown to improve the IVF process by reducing ROS production and peroxidative damage in the acrosome [49]. The results of two recent studies support this same role of RVT in the protection of IVF capacity. The antioxidant potential of RVT (to reduce the level of ROS and increase the level of glutathione) has been used to improve the maturation of spermatozoa and the IVF process in porcine oocytes [50]. Additionally, RVT has been shown to have a beneficial effect on the development of porcine embryos [51].

\section{Conclusions}

In conclusion, the present study provides evidence that RVT is a potent antioxidant in vitro. RVT was shown to protect spermatozoa from oxidative damage caused by ferrous iron/ascorbate through protection from lipid peroxidation and preservation of the IVF process.

\section{Conflicts of Interest}

The authors declare no conflict of interest.

\section{References}

1. Storey, K.B. Oxidative stress: Animal adaptations in nature. Braz. J. Med. Biol. Res. 1996, 29, 1715-1733.

2. Sanocka, D.; Kurpisz, M. Reactive oxygen species and sperm cells. Reprod. Biol. Endocrinol. 2004, 2, 1-7.

3. Bansal, A.K.; Bilaspuri, G.S. Impacts of oxidative stress and antioxidants on semen functions. Vet. Med. Int. 2011, 2011, doi:10.4061/2011/686137.

4. Kopers, A.J.; de luliis, G.N.; Finnie, J.M.; McLaughlin, E.A.; Aitken, R.J. Significance of mitochondrial reactive oxygen species in the generation of oxidative stress in spermatozoa. Endocr. Res. 2008, 93, 3199-3207.

5. Aitken, R.J.; Baker, M.A. Oxidative stress, sperm survival and fertility control. Mol. Cell. Endocrinol. 2006, 250, 66-69.

6. Agarwal, A.; Gupta, S.; Sikka, S. The role of free radicals and antioxidants in reproduction. Suresh Curr. Opin. Obstet. Gynecol. 2006, 18, 325-332. 
7. Tremellen, K. Oxidative stress and male infertility a clinical perspective. Hum. Reprod. Update 2008, 14, 243-258.

8. Kovacic, P.; Somanathan, R. Multifaceted approach to resveratrol bioactivity: Focus on antioxidant action, cell signaling and safety. Oxid. Med. Cell. Longev. 2010, 3, 86-100.

9. Bhat, K.; Kosmeder, J., II; Pezzuto, J. Biological effects of resveratrol. Antioxid. Redox Signal. 2001, 3, 1041-1064.

10. Cottart, C.; Antoine, V.; Morizot, C.; Beaudeux, J. Resveratrol bioavailability and toxicity in humans. Mol. Nutr. Food Res. 2010, 54, 7-16.

11. Xia, E.; Den, G.; Guo, Y.; Li, H. Biological activities of polyphenols from grapes. Int. J. Mol. Sci. 2010, 11, 622-646.

12. Juan, M.E.; González-Pons, E.; Munuera, T.; Ballester, J.; Rodríguez-Gil, J.E.; Planas, J.M. Trans-resveratrol, a natural antioxidant from grapes, increases sperm output in healthy rats. J. Nutr. 2005, 135, 757-760.

13. Shin, S.; Hee, J.J.; Park, D.; Jang, M.J.; Choi, J.H.; Choi, B.H.; Joo, S.S.; Nahm, S.S.; Kim, J.; Kim, Y.B. Trans-resveratrol relaxes the corpus cavernosum ex vivo and enhances testosterone levels and sperm quality in vivo. Arch. Pharm. Res. 2008, 31, 83-87.

14. Revel, A.; Raanani, H.; Younglai, E.; Xu, J.; Han, R.; Savouret, J.F.; Casper, R.F. Resveratrol, a natural aryl hydrocarbon receptor antagonist, protects sperm from DNA damage and apoptosis caused by benzo(a)pyrene. Reprod. Toxicol. 2001, 15, 479-486.

15. Uguralp, S.; Mizrak, B.; Bay Karabulut, A. Resveratrol reduces ischemia reperfusion injury after experimental testicular torsion. Eur. J. Pediatr. Surg. 2005, 15, 114-119.

16. World Health Organization (WHO). Laboratory Manual for the Examination of Human Semen and Semen Cervical Mucus Interaction; Cambridge University Press: Cambridge, UK, 2010.

17. Buege, J.A.; Aust, S.D. Microsomal lipid peroxidation. Metheods Enzymol. 1978, 52, 302-310.

18. Aitken, R.J.; Curry, B.J. Redox regulation of human sperm function: From the physiological control of sperm capacitation to the etiology of infertility and DNA damage in the germ line. Antioxid. Redox Signal. 2011, 14, 367-381.

19. Ford, W.C. Regulation of sperm function by reactive oxygen species. Hum. Reprod. Update 2004, 10, 387-399.

20. Griveau, J.F.; Dumont, E.; Renard, P.; Callegari, J.P.; Le Lannou, D. Reactive oxygen species, lipid peroxidation and enzymatic defence systems in human spermatozoa. J. Reprod. Fertil. 1995, 103, 17-26.

21. Buffone, M.G.; Ijiri, T.W.; Cao, W.; Merdiushev, T.; Aghajanian, H.K.; Gerton, G.L. Heads or tails? Structural events and molecular mechanisms that promote mammalian sperm acrosomal exocytosis and motility. Mol. Reprod. Dev. 2012, 79, 4-18.

22. Lavranos, G.; Balla, M.; Tzortzopoulou, A.; Syriou, V.; Angelopoulou R. Investigating ROS sources in male infertility: A common end for numerous pathways. Reprod. Toxicol. 2012, 34, 298-307.

23. Agarwal, A.; Tamer, M.S. Role of sperm chromatin abnormalities and DNA damage in male infertility. Hum. Reprod. Update 2003, 9, 331-345.

24. Zini, A.; San Gabriel, M.; Baazeem, A. Antioxidants and sperm DNA damage: A clinical perspective. J. Assist. Reprod. Genet. 2009, 26, 427-432. 
25. Zini, A.; Al-Hathal, N. Antioxidant therapy in male infertility: Fact or fiction? Asian J. Androl. 2011, 13, 374-381.

26. Lombardo, F.; Sansone, A.; Romanelli, F.; Paoli, D.; Gandini, L.; Lenzi A. The role of antioxidant therapy in the treatment of male infertility: An overview. Asian J. Androl. 2011, 13, 690-697.

27. Mínguez-Alarcón, L.; Mendiola, J.; López-Espín, J.J.; Sarabia-Cos, L.; Vivero-Salmerón, G.; Vioque, J.; Navarrete-Muñoz, E.M.; Torres-Cantero, A.M. Dietary intake of antioxidant nutrients is associated with semen quality in young university students. Hum. Reprod. 2012, 27, 2807-2814.

28. Zareba, P.; Colaci, D.S.; Afeiche, M.; Gaskins, A.J.; Jørgensen, N.; Mendiola, J.; Swan, S.H.; Chavarro, J.E. Semen quality in relation to antioxidant intake in a healthy male population. Fertil. Steril. 2013, 100, 1572-1579.

29. Afeiche, M. Williams, P.L.; Mendiola, J.; Gaskins, A.J.; Jørgensen, N.; Swan, S.H.; Chavarro, J.E. Dairy food intake in relation to semen quality and reproductive hormone levels among physically active young men. Hum. Reprod. 2013, 28, 2265-2275.

30. Ourique, G.M.; Finamor, I.A.; Saccol, E.M.; Riffel, A.P.; Pês, T.S.; Gutierrez, K.; Gonçalves, P.B.; Baldisserotto, B.; Pavanato, M.A.; Barreto, K.P. Resveratrol improves sperm motility, prevents lipid peroxidation and enhances antioxidant defences in the testes of hyperthyroid rats. Reprod. Toxicol. 2013, 37, 31-39.

31. Yuluğ, E.; Türedi, S.; Alver, A.; Türedi, S.; Kahraman, C. Effects of resveratrol on methotrexate-induced testicular damage in rats. Sci. World J. 2013, 2013, doi:10.1155/2013/489659.

32. Goldberg, D.M.; Ng, E.; Yan, J.; Karumanchirí A.; Soleas, G.J.; Diamandis, E.P. Regional differences in resveratrol isomer concentrations of wines from various cultivars. J. Wine Res. 1996, 7, 13-24.

33. McMurtrey, K.D. Resveratrol in Wine. In Wine: Nutritional and Therapeutic Benefits; Watkins, T.R., Ed.; Oxford University Press: Oxford, UK, 1997; pp. 45-55.

34. Juan, M.E.; Vinardell, M.P.; Planas, J.M. The daily oral administration of high doses of trans-resveratrol to rats for 28 days is not harmful. J. Nutr. 2002, 132, 257-260.

35. Patel, K.R.; Scott, E.; Brown, V.A.; Gescher, A.J.; Steward, W.P.; Brown, K. Clinical trials of resveratrol. Ann. N. Y. Acad. Sci. 2011, 1215, 161-169.

36. Silva, E.C.; Cajueiro, J.F.; Silva, S.V.; Soares, P.C.; Guerra, M.M. Effect of antioxidants resveratrol and quercetin on in vitro evaluation of frozen ram sperm. Theriogenology 2012, 77, $1722-1726$.

37. Aitken, R.J.; Jane, S.; Clarkson, S.; Fishel, S. Generation of reactive oxygen species, lipid peroxidation, and human sperm function. Biol. Reprod. 1989, 40, 183-197.

38. Martínez-Pastor, F.; Aisen, E.; Fernández-Santos, M.R.; Esteso, M.C.; Maroto-Morales, A.; García-Álvarez, O.; Garde, J.J. Reactive oxygen species generators affect quality parameters and apoptosis markers differently in red deer spermatozoa. Reproduction 2009, 137, 225-235.

39. Buettner, G.R.; Jurkiewicz, B.A. Catalytic metals, ascorbate and free radicals: Combinations to avoid. Radiat. Res. 1996, 145, 532-541.

40. Armstrong, J.S.; Rajasekaran, M.; Chamulitrat, W.; Gatti, P.; Hellstrom, W.J.; Sikka, S.C. Characterization of reactive oxygen species induced effects on human spermatozoa movement and energy metabolism. Free Radic. Biol. Med. 1999, 26, 869-880. 
41. Leonard, S.S.; Xia, C.; Jiang, B.H.; Stinefelt, B.; Klandorf, H.; Harris, G.K.; Shi, X. Resveratrol scavenges reactive oxygen species and effects radical-induced cellular responses. Biochem. Biophys. Res. Commun. 2003, 309, 1017-1026.

42. Gravance, C.G.; Garner, D.L.; Miller, M.G.; Berger, T. Fluorescent probes and flow cytometry to assess rat sperm integrity and mitochondrial function. Reprod. Toxicol. 2001, 15, 5-10.

43. Van Ginkel, P.R.; Sareen, D.; Subramanian, L.; Walker, Q.; Darjatmoko, S.R.; Lindstrom, M.J.; Kulkarni, A.; Albert, D.M.; Polans, A.S. Resveratrol inhibits tumor growth of human neuroblastoma and mediates apoptosis by directly targeting mitochondria. Clin. Cancer Res. 2007, 13, 5162-5169.

44. Zini, R.; Morin, C.; Bertelli, A.; Bertelli, A.A.; Tillement, J.P. Effects of resveratrol on the rat brain respiratory chain. Drugs Exp. Clin. Res. 1999, 25, 87-97.

45. Collodel, G.; Federico, M.G.; Geminiani, M.; Martini, S.; Bonechi, C.; Rossi, C.; Figura, N. Effect of trans-resveratrol on induced oxidative stress in human sperm and in rat germinal cells. Reprod. Toxicol. 2011, 31, 239-246.

46. Berrougui, H.; Grenier, G.; Loued, S.; Drouin, G.; Khalil, A. A new insight into resveratrol as an atheroprotective compound: Inhibition of lipid peroxidation and enhancement of cholesterol efflux. Atherosclerosis 2009, 207, 420-427.

47. López-Vélez, M.; Martinez-Martinez, F.; Del Valle-Ribes, C. The study of phenolic compounds as natural antioxidants in wine. Crit. Rev. Food Sci. Nutr. 2003, 43, 233-244.

48. Visconti, P.E.; Ning, X.; Fornes, M.W.; Alvarez, J.G.; Stein, P.; Connors, S.A.; Kopf, G.S. Cholesterol efflux-mediated signal transduction in mammalian sperm: Cholesterol release signals an increase in protein tyrosine phosphorylation during mouse sperm capacitation. Dev. Boil. 1999, 214, 429-443.

49. Gray, J.E.; Starmer, J.; Lin, V.S.; Dickinson, B.C.; Magnuson, T. Mitochondrial hydrogen peroxide and defective cholesterol efflux prevent in vitro fertilization by cryopreserved inbred mouse sperm. Biol. Reprod. 2013, 89, 17.

50. Kwak, S.S.; Cheonga, S.A.; Jeona, Y.; Leeb, E.; Choic, K.C.; Jeungc, E.B.; Hyun, S.H. The effects of resveratrol on porcine oocyte in vitro maturation and subsequent embryonic development after parthenogenetic activation and in vitro fertilization. Theriogenology 2012, 78, 86-101.

51. Lee, K.; Wang, C.; Chaille, J.M.; Machaty, Z. Effect of resveratrol on the development of porcine embryos produced in vitro. J. Reprod. Dev. 2010, 56, 330-335.

(C) 2014 by the authors; licensee MDPI, Basel, Switzerland. This article is an open access article distributed under the terms and conditions of the Creative Commons Attribution license (http://creativecommons.org/licenses/by/3.0/). 